\title{
Ribotypes in isolates and testing algorithm of C. difficile infections in the studied sample
}

\author{
Stofkova $Z^{1}$, Novakova $E^{1}$, Novak $M^{2}$ \\ Institute of Microbiology and Immunology, Comenius University in Bratislava, Jessenius Faculty \\ of Medicine in Martin. zuzana.stofkova@gmail.com
}

\begin{abstract}
BACKGROUND: Clostridium (Clostridioides) difficile is the most common pathogen of nosocomial and antibiotic-related diarrhea in health-care facilities. The aim of the analysis was to show the testing algorithm and to identify hypervirulent strains (suspected RT 027).

METHODS: The retrospective analysis of patient samples suspected on CDI was carried out by a two-step algorithm. Biological specimens were analysed by GDH or culture, immunoenzymatic assay on toxins A/B and selected samples also by a real-time PCR.

RESULTS: In 1006 specimen suspected on CDI, 202 specimens were evaluated as positive in the twostep algorithm. Conflicting results (64 C. difficile isolates) were tested in a three-step algorithm by a realtime PCR and revealed 59 toxigenic and non RT 027 ribotypes. Statistically significant dependence among the independent variables, such as: diagnostic parameters and length of hospitalization $(p=0.175)$ and $C$. difficile (suspected RT027) ribotypes was not found.

CONCLUSION: The results of PCR ribotyping showed a high prevalence of hypervirulent and toxigenic ribotypes in the studied sample. A resistance to vancomycin was found in one isolate. The PCR method contributed to the rapid laboratory diagnosis and thus treatment of high risk patients or was used as a third step in in the case of unclear results of standard diagnostic methods
\end{abstract}

(Tab. 1, Fig. 4, Ref. 18). Text in PDF www.elis.sk.

KEY WORDS: toxigenic $C$. difficile, diagnostics, real-time PCR, ribotyping.

\section{Introduction}

Toxigenic strains of $C$. difficile are among the most common inducers of diarrhea acquired in the hospital environment and are considered to be the cause of a large proportion of antibiotic-related nosocomial diarrhea. Over the last two decades, there has been an increase in the incidence of CDI cases and severity of CDI infections, and new highly virulent $C$. difficile strains (e.g. ribotype 027) emerged (1).

An accurate and fast diagnostics of CDI is essential for an optimal patient care and to prevent the spread of infection (2).

C. difficile produce 2 large toxins. Toxin $\mathrm{A}$ is an enterotoxin and toxin $\mathrm{B}$ is a cytotoxin. C. difficile can produce a third toxin, referred to as the "binary toxin" (3). However, there are strains of C. difficile, which do not produce any of the toxins and are unable to induce the disease (4).

\footnotetext{
${ }^{1}$ Institute of Microbiology and Immunology, Comenius University in Bratislava, Jessenius Faculty of Medicine in Martin, Slovakia, and ${ }^{2}$ Department of Public Health, Comenius University in Bratislava, Jessenius Faculty of Medicine in Martin, Slovakia

Address for correspondence: $\mathrm{Z}$. Stofkova MD, PhD, Institute of Microbiology and Immunology, Comenius University in Bratislava, Jessenius Faculty of Medicine in Martin, Mala Hora 11149/4B, SK-036 01 Martin, Slovakia.

Phone: +421904517772
}

In order to manage $C$. difficile infections effectively, a rapid and accurate diagnostics is essential to guide the treatment and to prevent its transmission.

The diagnosis of CDI is based on a combination of symptoms confirmed by a microbiological evidence of toxins produced by $C$. difficile or toxigenic strains of $C$. difficile in faeces, respectively colonoscopic evidence, or in the absence of other causes (5).

The optimal diagnostic approach to CDI is still under discussion. According to ESCMID (European society for clinical microbiology), the use of one standalone CDI test is not recommended due to the low positive predictive value at low CDI prevalence (2).

Due to the different sensitivity of the different methods used, according to current recommendations, a combination of at least two different tests is preferred. Therefore, at least 2 step algorithms are currently used to optimize CDI diagnostics (6).

In $2013,48 \%$ of hospitals in 20 European countries were using an optimised algorithm for laboratory diagnosis of CDI. Two-stage algorithms using a glutamate dehydrogenase enzyme immunoassay (EIA) or NAAT (e.g. real-time PCR) followed by a toxin detection have been adopted in the UK (1)

Testing algorithm initiated by PCR method followed by toxin (EIA), e.g. ELISA method is not yet a part of routine practice in many countries (7).

Diagnostic methods for identification of different target regions determine the presence of free toxins or toxigenic strains. 
Methods that determine the presence of free faeces toxins are: immunoenzymatic assay (EIA) and cytotoxicity and neutralisation assay (CTNA). Methods that detect the presence of $C$. difficile include: EIA assay for evidence of glutamate dehydrogenase enzyme (GDH) and anaerobic culture and methods that detect the presence of a toxigenic C. difficile include: toxigenic culture (TC) with a high clinical sensitivity or NAAT (e.g. „real-time“ PCR) method. A standard test for the detection of toxins (evidence of cytopathic effect on cell cultures and neutralization assay - CTNA or CCCNA) and toxigenic culture are used as the reference methods for laboratory confirmation of CDI (2).

The first test should have a high negative predictive value of NPV (i.e. a highly sensitive test that reliably excludes patients without CDI. This may be an immunoenzyme assay for glutamate dehydrogenase or a PCR assay. In the positive result, the second test with a high positive predictive value, i.e. a highly specific test, such as A / B toxin detection is carried out (5). If the GDH test was performed as the first test in sequence, it is possible to carry out PCR screening as the third step. EIA for toxins - immunoenzymatic methods based on the detection of toxin $\mathrm{A} / \mathrm{B}$ are considered to be less sensitive at the detection of toxigenic $C$. difficile than the PCR method (6). The importance of the realtime PCR method lies in the high sensitivity and specificity of the testing method and therefore can be used to accelerate the exclusion of C. difficile infection (7). The testing algorithm represents potential cost savings for laboratories. Each laboratory must evaluate and assess a multi-stage algorithm for its patient population (6).

\section{Materials and methods}

The samples were analysed in the Laboratory in Žilina (Klinicka biochemia). The data were analysed retrospectively from 1.1.2015 - 30.6.2016 by the following laboratory methods. The patient's specimens were tested by direct diagnostic methods for C. difficile using immunochromatographic assay for the detection of Glutamate dehydrogenase enzyme (CERTEST Clostridium difficile GDH), toxins A or B (CERTEST Clostridium difficile Toxin A / B), and immunoenzymatic methods ELISA (ProSpectT C.diffcile Toxin A / B Microplate assay) to determine toxins A and B. Selected biological samples were analyzed also by multiplex real-time PCR (GeneXpert, Cepheid). The anaerobic cultivation on taurocholate-cycloserine-cefoxitin-fructose agar was established to confirm positive C. difficile assays. The MALDI TOF method was used to identify C. difficile on species und subspecies level.

\section{C. difficile culture and identification by MALDI-TOF MS}

The samples were directly cultured on selective media. The samples were inoculated into cycloserin-cefoxitin-fructose agar, supplemented with taurocholate and incubated under anaerobic conditions at $37{ }^{\circ} \mathrm{C}$ for 72 hours. C. difficile was identified by Matrix Assisted Laser Desorption/Ionisation Time-of-Flight Mass Spectrometry (MALDI-TOF MS) with the use of MALDI Biotyper v 3.0 system (Brucker Daltonics).

\section{Antibiotic susceptibility testing of $C$. difficile isolates}

C. difficile isolates were tested to vancomycin and metronidazole using E-test (BioMerieux) on blood agar (Oxoid). The minimum inhibitory concentration (MIC - minimal concentration that stops the growth of the bacterium) breakpoints for metronidazole and vancomycin were applied according to European Committee on Antimicrobial Susceptibility testing (EUCAST).

The detection of toxins was carried out by multiplex „realtime " PCR method (GeneXpert C. difficile Epi PCR Cepheid, Inc., $\mathrm{CA}$ ) - enables the detection of the B toxin gene (tcdB), the binary toxin genes (cdtA a cdt B), and deletion of tcdC gene on nucleotide 117 was carried out, which is suspicious for RT 027. Capillary electrophoresis PCR ribotyping is needed to be performed by amplification of ribosomal DNA and their separation.

Patient file results were processed using Excel (Microsoft), then the selected file was exported to SPSS, a 2-tailed t-test was used for the normal distribution of metric data. The Chi square test as well as the Fisher test for smaller file sizes were applied.

\section{Results}

The group of 1006 samples suspected for CDI was analysed from January 2015 to June 2016 by different testing methods. The number of patients' samples tested positively for CDI from a total number of 1006 samples was 148. The samples from patients evaluated positively in immunoenzymatic assay for A / B toxins and culture/GDH and the total number of positively tested samples by the 2 -step algorithm are shown in Figure 1.

148 patients' samples were in $43 \%$ from male and $57 \%$ were from female patients. The majority of the positively tested samples were from patients over 65 years $(76 \%)$ and $24 \%$ of the samples were from patients under 65 years.

According to the algorithm, $63 \%$ of samples (633 samples) were evaluated as negative by GDH test or culture, which excluded the presence of $C$. difficile in specimen (Additionally, detection of toxins $\mathrm{A} / \mathrm{B}$ was also negative). 373 samples were detected for GDH enzyme with the positive result and according to the 2-step algorithm were analysed further for detection of toxins A/B. $18 \%$ of samples (183 samples) were evaluated positively for toxins A/B by EIA, with a high probability of CDI.

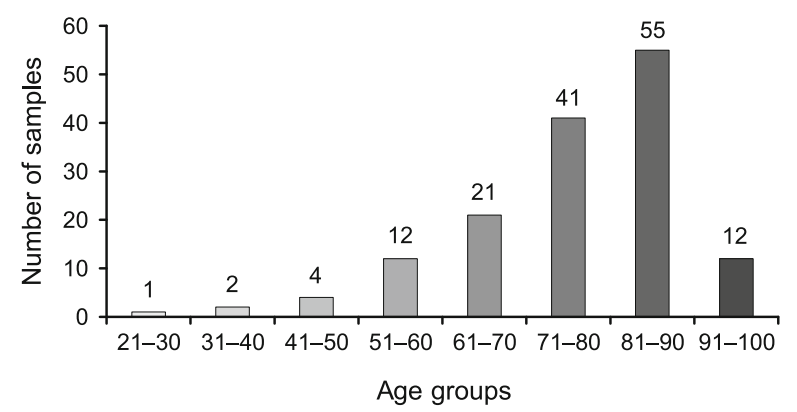

Fig. 1. Positive patients' samples by GDH/culture and toxins A/B (EIA) according to the age groups in 2015-2016. 


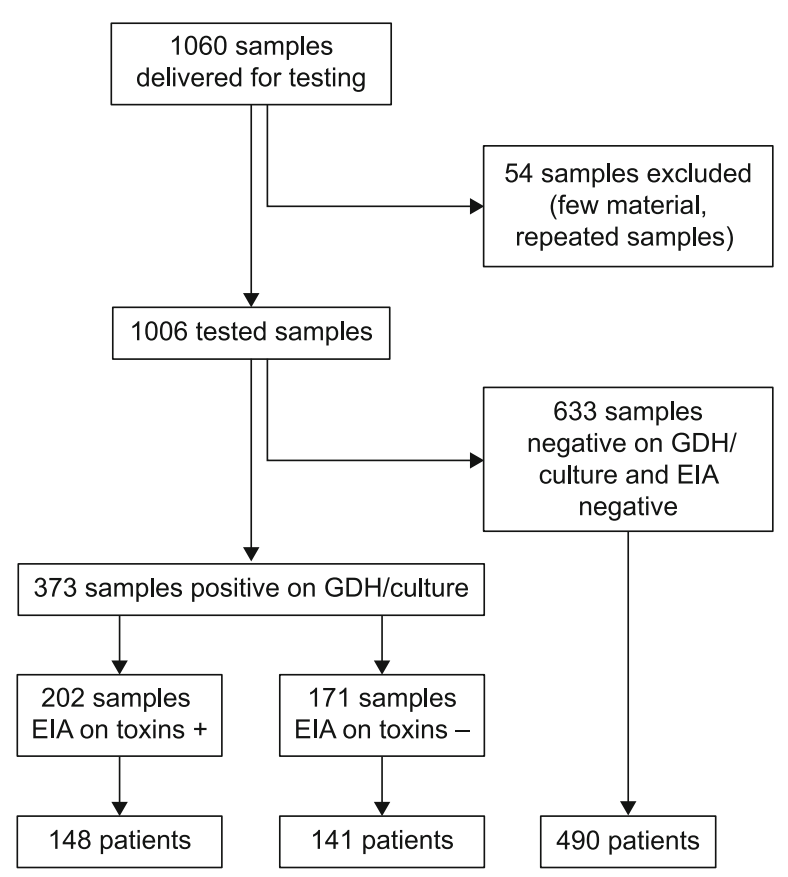

Fig. 2. Testing algorithm on CDI in the laboratory in 2015-2016.

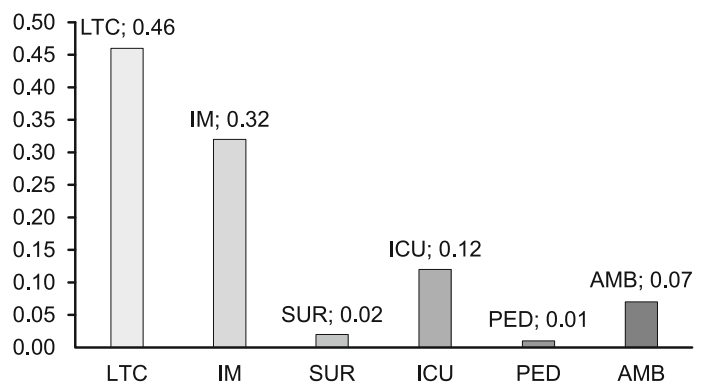

Fig. 3. Total number of positive samples in $\%$ of total samples from patients within departments (2-step algorithm, GDH test or anaerobic culture and ELISA test).

$19 \%$ of samples (191) were detected as GDH positive and toxin $\mathrm{A} / \mathrm{B}$ negative by EIA test. This could be carriers of toxigenic strain/s or the amount of toxin was not sufficient amount to be clearly detected in an immunoenzymatic test. The preanalytic phase conditions (toxins are termolabile) could also play the role (Fig. 2).

From 171 samples, 64 samples with conflicting results and with clinical signs of suspected CDI were evaluated by the realtime PCR. Real-time PCR was carried out as the third step in the diagnostic algorithm.

The samples positively tested were from hospitalised patients (- $93 \%$ from internal departments and departments of long term care and from non-hospitalised patients $-7 \%$ ) (Fig. 3).

The samples most frequently positively tested were from internal departments, representing up $32 \%$ of the total samples tested. Positive samples from the long-term care departments comprised $46 \%$ of the total samples tested, followed by positive samples from anesthesiology - resuscitation department $12 \%$ and samples from surgery departments $3 \%$, and ambulant patients $7 \%$ (Fig. 3).

Genotypic and phenotypic characteristics of C. difficile in the studied sample

We analysed a set of 64 samples on toxins by immunochromatography for toxins $\mathrm{A} / \mathrm{B}$ and $\mathrm{GDH}$, real-time PCR, enzyme immunoassay, and culture. 59 samples were tested positive and 5 negative. The mean age was 77 years, 75 years for male and 79 for women. 17 isolates were tested by culture. The patients' samples with the need of rapid testing or with conflicting results on GDH and toxins $\mathrm{A} / \mathrm{B}$ (GDH positive and toxins $\mathrm{A} / \mathrm{B}$ negative or slightly positive) were tested with the ,real-time“ $\mathrm{PCR}$ method in order to exclude the presence of toxigenic strain.

64 samples were from internal department patients (34 samples), department of long term care -24 and surgery department -1 , intensive care unit -1 and from ambulant patients -4 . The length of hospitalization of the patients was on average 46 days.

From 64 C. difficile isolates included in the studied sample 16 carried the B toxin gene (evaluated as toxigenic), 43 isolates carried both the B toxin gene and the binary toxin genes, and the deletion of nucleotide 117 on the tcdC regulatory gene for $\mathrm{B}$ toxin (hypervirulent or supertoxigenic) and $5 \mathrm{C}$. difficile isolates were negative (Tab. 1). In further surveillance procedure, the samples were sent for capillary electrophoresis PCR ribotyping detection.

Representation of ribotypes within the sample were as follows: suspected ribotypes RT027, and toxigenic ribotypes (non RT027). Within internal department, toxigenic ribotypes (non RT027) comprised -8 isolates, suspected RT027 - 24 isolates, 2 were negative. Department of long-term care: suspected RT027 (16) and toxigenic ribotypes (non RT027) (7), 1 sample was negative. Surgery department -1 isolate was non RT027, Intensive care unit (ICU) - 1 negative isolate, ambulant -2 isolates suspected RT027, 1 isolate nonRT027, 1 negative.

Meanwhile, these ribotypes were present in patients' specimen from in-patient departments (internal departments, departments of long-term care) but also in outpatients, which had a history of prior hospitalization. It shows the circulation of the toxigenic and hypervirulent ribotypes among the departments. Most of the patients were hospitalised several times with chronical diseases and they were further treated at the long-term care department.

The C. difficile isolates were tested on antimicrobial susceptibility on vancomycin and metronidazole. Minimal inhibition concentration (MIC 8-32 $\mu \mathrm{g} / \mathrm{ml}$ ) breakpoints were evaluated according to the standards of the European committee for susceptibility testing (EUCAST). The importance of anaerobic cultivation resides in the determination of $C$. difficile susceptibility on antimicrobial agents. The isolates $C$. difficile in the studied sample were susceptible to metronidazole (MTZ) and vancomycin (VAN). One $C$. difficile isolate was resistent to vancomycin (Tab. 1).

In the selected sample of patients, further indicators (creatinine, albumin, CRP, length of hospitalization) were compared between the patients with different ribotypes (RT027 presumptive positive and non RT027) in samples. There was no statistically significant 
Tab. 1. Genotype and fenotype characteristics of $C$. difficile isolates in the studied samples.

\begin{tabular}{|c|c|c|c|c|c|c|}
\hline $\begin{array}{l}\text { Isolates of } \\
\text { C. difficile }\end{array}$ & $\begin{array}{r}\text { Fenotyp } \\
\text { of } C . d\end{array}$ & $\begin{array}{l}\text { cteristics } \\
\text { solates }\end{array}$ & & otype characteristi & s of $C$. difficile isolc & \\
\hline Number of isolates & $\begin{array}{l}\mathrm{MIC} \mu \mathrm{g} / \mathrm{ml} \\
\text { (VAN) }\end{array}$ & $\begin{array}{c}\mathrm{MIC} \mu \mathrm{g} / \mathrm{ml} \\
(\mathrm{MTZ})\end{array}$ & $\begin{array}{l}\text { gene for B toxin } \\
(\text { tcdB })\end{array}$ & $\begin{array}{l}\text { genes cdtA, cdtB } \\
\text { for binary toxin }\end{array}$ & $\begin{array}{c}\text { deletion of nt } 117 \\
\text { in tcdC gene } \\
\text { (susp. RT 027) }\end{array}$ & $\begin{array}{c}\text { PCR ribotypes } \\
\text { Presumptive } \\
\text { RT027 }\end{array}$ \\
\hline 15 & sensitive & sensitive & positive & negative & negative & negative \\
\hline 1 & resistant & sensitive & positive & negative & negative & negative \\
\hline 43 & sensitive & sensitive & positive & positive & positive & positive \\
\hline 5 & sensitive & sensitive & negative & negative & negative & negative \\
\hline
\end{tabular}

dependence among the independent variables, such as creatinine $(\mathrm{p}=0.524)$, albumin $(\mathrm{p}=0.682), \mathrm{CRP}(\mathrm{p}=0.295)$ and the length of hospitalization $(\mathrm{p}=0.175)$ among PCR ribotypes (non RT027 suspected) (e.g.RT001,etc.) and ribotypes (presumptive RT027 - e.g. RT176).

We can conclude that the laboratory results in patients with ribotype RT027 and patients with non ribotype RT027 did not show a significant difference. We explain this by the size of the sample (limited size of patients) and patients with multiple co-morbidities, such as: cardiovascular diseases, lung diseases, renal diseases and oncological diseases.

In the analysed procedure, we tested the samples for GDH and toxins A/B (EIA) - two-step algorithm. The samples GDH (EIA) positive and toxins $\mathrm{A} / \mathrm{B}$ negative underwent testing for ELISA test and subsequent PCR testing was performed as the third step, as shown in the Figure 4.

Interpretation of the test results - if GDH (immunoenzymatically) or PCR positive and toxin ELISA positive (PPV $=91.4 \%$ ), then C. difficile is most likely present. The result should be compulsorily reported. If GDH (immunoenzymatically) or PCR is positive and the toxin ELISA negative, then C. difficile may be present, i.e. potentially a carrier of $C$. difficile - results are not included in the mandatory report. If GDH (immunoenzymatically) or PCR negative toxin by ELISA is negative (NPV $=98.9 \%$ ), then C. difficile is very unlikely to be present (7).

\section{Discussion}

C. difficile is an anaerobic gram-positive spore-forming bacillus. Colonization rates in healthy humans in the community range from $0.8 \%$ to $13 \%$ and are higher in long-term care facility residents (8) This frequency is higher in hospitalised patients (20-30 $\%$ ). Colonization with $C$. difficile does not automatically lead to development of symptomatic CDI (2).

Transmission is by oral-fecal route. Intestinal dysmicrobia (most commonly associated with antibiotic therapy) may be considered to be the most important factor of infection, which may result in a loss of colonisation resistance to C. difficile.

The immune response of the host may partially explain how colonisation with $C$. difficile results in a wide spectrum of outcomes (8), such as diarrhea, colitis or pseudomembranous colitis with complications. Risk factors for severe CDI are: age over 65 years, treatment with broad spectrum antibiotics, long-term hospitalization (longer than 3 weeks), major underlying disease (4)

It has been shown that a rapid diagnostics positively impacts the patient's care by reducing delays in the initiation of the isolation and treatment for the confirmed CDI cases (2).

The incidence of CDI in Slovakia has a growing trend, with incidence $19.5 / 10,000$ patients in 2016. In addition to the fact that the incidence of CDI in the SR is increasing, it is mainly due to better reporting and surveillance that helps to monitor CDI cases.

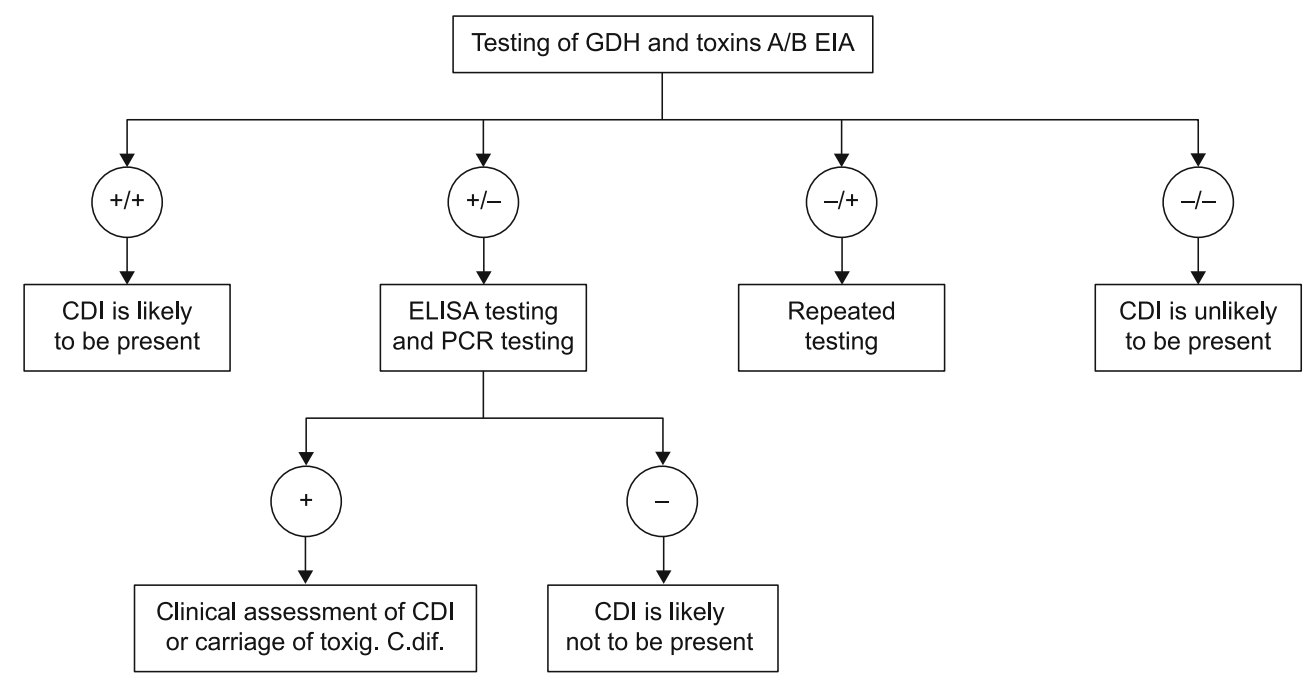

Fig.4. Testing protocol (algorithmic approach) adopted in the laboratory practice (own processing according (2) and (5). 
The number of reported cases of CDI in medical institutions (HAICDI) in Slovakia reached within internal wards $88.58 \%$, followed by surgical wards, intensive care unit (ICU) and others (9).

These finding were also confirmed by our positively evaluated samples, which came mainly from hospitalised patients (93\%) (from which were $78 \%$ internal departments and departments of long-term care) and non-hospitalised patients (7\%).

Our results also revealed that the majority of positively tested CDI cases are with the age over 65 years $-75 \%$ of all cases.

The real-time PCR can help an early diagnostics and early recognition of patients with $C$. difficile before complications occur. The PCR tests are very sensitive to C. difficile, but do not distinguish between an active infection and asymptomatic colonisation as they determine the genes for production of toxins (6). Genetic evidence of the toxigenic strain does not automatically mean the toxigenic genes' expression.

PCR detected a possible deletion of tcdC, a suspected ribotype 027 , which was important from the epidemiological point of view, but also the turn-around time and thus early treatment. Not only would the patient benefit from this, but the possible cost of patient care in the event of complications would also be reduced ( 7 ).

Traditional typing methods used to identify the ribotype of a strain causing CDI required culture first and so were not timely. In this regard, a rapid ,presumptive RT027“" could be of value. The assay had a high NPV for ,presumptive RT027, but did overcall the number of samples that truly contained PCR-ribotype 027 $(69.9 \%)(10)$.

Samples with conflicting results between the highly sensitive GDH assay and the A and B toxins that had a lower sensitivity but higher specificity were often discussed. Studies recommend using PCR for such discrepancies.

In cases suspected of CDI (GDH positive, toxine negative) - the real-time PCR method can confirm or exclude a presence of toxigenic strain, even if it does not differentiate between the symptomatic infection and colonisation. (7).

Notably, outcomes of the patients, who are NAAT positive and toxin negative, are indistinguishable from NAAT negative and toxine negative (10).

For patients, who are C. difficile positive by molecular-biological assay but, who are toxin negative by immunoenzymatic methods, e.g. ELISA method, may be referred to as "C. difficile" carriers, although they may be isolated but do not require treatment (7).

The hypervirulent PCR ribotype 027 is known worldwide. Some studies point to a more severe course of CDI disease, when this ribotype occurs. The hypervirulent strain is referred to as $C$. difficile BI / NAP1 / 027 (6). The strain BI / NAP1 / 027 contains a nucleotide mutation at position 117 on the tcdC gene that encodes the protein $\mathrm{C}$, which causes the suppression of genes for A/B toxins (11).

Further analyzes showed that there are more ribotypes with similar properties (4).

Ribotype 176 is close to ribotype 027 and also encodes binary toxin and contains a nucleotide mutation at position 117 on the
tcdC gene that encodes the protein $\mathrm{C}$, that causes the suppression of genes for $\mathrm{A} / \mathrm{B}$ toxins, and can be erroneously identified by commercial assays aimed at deletion of one base pair at nucleotide 117 in the $C$. difficile tcdC gene.

This ribotype persists in the Czech Republic and has been recorded in Poland in 2013 (12). According to studies (13) it is also present in Slovakia.

It is important to note, that there may be other attributes of the $C$. difficile genome that can significantly affect virulence (not only binary toxin and tcdC deletion) and hence the clinical course of the disease, which should be taken into account in treatment strategy management (14).

The ribotype or strain type is referred to as another possible cause of increased morbidity, severity of disease and higher CDI relapse rates. According to the Canadian study conducted by Miller, PCR ribotype 027 correlated with an increased disease severity in patients, in almost all age groups. However, toxin expression is related to CDI severity. Several studies report a high and uncontrolled toxin production for ribotype 027 (i.e. deletion of negative control tcdC gene).

The most frequently occurring ribotypes in our region according to the study published by Novaková et al. were RT176 (among hypervirulent ribotypes-presumptive RT027) and 001 among toxigenic ribotypes (non RT027) (13).

Among toxigenic ribotypes we distinguish 15 toxinotypes (I - XV) (15).

Hypervirulent ribotype 176 is also associated with a more severe course of the disease and may be misidentified by commercial assays aimed at deletion on base pair at nucleotide 117 in the tcdC regulatory gene for B toxin (4) We suppose this fact also in our study sample (13).

The new epidemic strains are less sensitive to antibiotics, e.g. resistance to fluoroquinolones. For many strains, metronidazole susceptibility decreases gradually, which can be demonstrated by an increasing MIC (minimal inhibitory concentration).

Epidemic ribotypes were connected with multiple antimicrobial resistance. Ribotypes 017,018 a 356 showed a high level of antimicrobial resistance (16).

This was confirmed also in our studied sample, one isolate showed a resistance to vancomycin.

PCR ribotyping is useful for monitoring the spread of CDI, the course of disease, as well as the detection of resistance to antimicrobial agents. This method is performed to identify individual strains, to carry out surveillance of the $C$. difficile infection spreading.

Despite an increased virulence of certain ribotypes, the PCR ribotype value as a predictor of disease severity is limited because the ribotype involved in infection is not known until it is diagnosed. However, in PCR epidemics, the ribotype could be considered, when deciding on the choice of empirical treatments (17).

In the recent extensive study (18) it was shown that the effect of individual ribotypes on overall disease progression and mortality and biomarkers (especially those associated with inflammation) varied. In addition to $C$. difficile PCR ribotype 027 , there are other strains that are associated with epidemics and a severe course of C. difficile infection. 
It is necessary to provide a timely appropriate antibiotic therapy with an early selection of patients who are at high risk and to perform a rapid selection of the most appropriate therapy. Predictive markers associated with a poor prognosis of CDI (e.g. hemodialysis, intubation, etc.), laboratory predictive markers, such as: differential blood count, acute phase protein levels, procalcitonin, lactate level, calprotectin, which are significant predictive factors as well as parameters that comprehensively assess water - mineral metabolism (4).

At the same time, the interpretation of the results requires the knowledge of the possible limits of specific diagnostic methods and experience in the clinical assessment of the results of specific diagnostic methods in clinical microbiology.

Reliable data is also crucial for monitoring CDI incidence over time and comparing individual healthcare facilities.

The treatment and diagnostics of the disease laboratory findings should always be interpreted together with patient status and other laboratory results, diagnostic methods and the patient's clinical status as well as the current epidemiological situation (4).

\section{Conclusion}

C. difficile infection (CDI) is the most common pathogen of nosocomial and antibiotic-related diarrhea in health care facilities and a significant medical and economic burden in healthcare institutions. Accurate and fast diagnostics of CDI is essential for an optimal patient care and in order to prevent the spread of infection.

For the treatment and diagnostics of disease, microbiological laboratory diagnosis 2-step algorithm was applied involving a high sensitivity screening assay (GDH EIA), followed by a high specificity assay (EIA for toxins A/B) is important. The 3 - step algorithm was applied in the diagnostic procedure. Conflicting results were tested by the „real-time“ PCR. The „real-time“ PCR method can assist in the early diagnosis and early recognition of patients with $C$. difficile infection before complications occur. GDH and the „real-time“ PCR test have a high NPV and can therefore be used to accelerate the exclusion of $C$. difficile infection.

PCR ribotyping identified 2 different PCR ribotypes (suspected RT027) in 43 isolates and 16 isolates (non RT027). The ribotype RT 176 is genetically close to ribotype 027 , it has a deletion in nucleotide 117 , in regulation gene (tcdC) for toxin B. The ribotype 001 and ribotype 176 with a high prevalence are the most present PCR ribotypes in our country.

C. difficile isolates were tested for susceptibility to metronidazole and vancomycin. One isolate (non RT027) was resistant to vancomycin. The retrospective analysis of patient samples suspected for CDI was carried out from January 2015 to July 2016.

The real-time PCR method contributes to the rapid diagnostics of high-risk patients in case of unclear results of standard phenotypic methods directed on exoenzyme (GDH EIA), and toxins (EIA). PCR ribotyping plays also an important role in CDI surveillance.

\section{References}

1. Davies KA, Longshaw CM, Davis GL et al. Underdiagnosis of Clostridium difficile across Europe: the European, Multicentre, Prospective, Biannual, Point-Prevalence Study of Clostridium difficile Infection in Hos- pitalised Patients With Diarrhoea (EUCLID). Lancet Infect Dis 2014; 14 (12): 1208-1219.

2. Gateau C, Couturier J, Coia J, Barbut F. How to: diagnose infection caused by Clostridium difficile. Clin Microbiol Infect 2018; 24 (5): 463-468.

3. Perelle S, Gilbert M, Bourlioux P, Corthier G, Popoff M.R. Production of a complete binary toxin (actin-specific ADP-ribosyltransferase) by Clostridium difficile CD196. InfectImmun 1997; 65: 1402-1407.

4. Jarčuška $P$ et al. Odporúčaný postup diagnostiky a liečby kolitídy spôsobenej Clostridium difficile. Via pract 2015; Suppl 1: 12 (S1).

5. Crobach MJT, Planche T, Eckert $\mathrm{C}$ et al. European Society of Clinical Microbiology and Infectious Diseases: update of the diagnostic guidance document for Clostridium difficile infection. Clin Microbiol Infect 2016; 22 (Suppl 4): S63-81.

6. Burnham CA, Carroll KC. Diagnosis of Clostridium difficile Infection: an Ongoing Conundrum for Clinicians and for Clinical Laboratories. Clin Microbiol Rev 2013; 26 (3): 604-630.

7. Robotham J, Wilcox M. Updated Guidance on the Diagnosis and Reporting of Clostridium Difficile. NHS, Advisory Committee on Antimicrobial Resistance and Healthcare Associated Infection (ARHAI), 2012. Available: https://www.gov.uk/government/uploads/system/uploads/attachment_data/ file/146808/dh_133016.pdf.pdf

8. Solomon K. The host immune response to Clostridium difficile infection. Ther Adv Infect Dis 2013; 1 (1): 19-35.

9. Novak M, Mazakova I, Sadlonova V, Cervenova T, Hudeckova H. Appearance of Clostridium Difficile infections in health care institutions in Slovakia and in the district of Martin. Acta Med Martin 2018; 18 (2): 5-12.

10. Davies, KE, Planche T, Wilcox MH. The predictive value of quantitative nucleic acid amplification detection of Clostridium difficile toxin gene for faecal sample toxin status and patient outcome. PLoS ONE 201813 (12): e0205941.

11. Brazier JS, Borriello SP. Microbiology, Epidemiology and diagnosis of Clostridium difficile. Curr Top Microbiol Immunol 2000; 250: 1-33.

12. Krutova M., Wilcox MH, Kuijper EJ. The pitfalls of laboratory diagnostics of Clostridium difficile infection. Clin Microbiol Infect 2018; 24 (7): 682-683.

13. Novaková E, Krutova M, Nyc O, Kuijper Ed J, Garabasova M, Novak M, Kotlebova N, Stefkovicova M. A high prevalence of Clostridium difficile ribotypes 001 and 176 recognized within an enhanced option of European standardized Clostridium difficile infection surveillance in Slovakia, 2016. 28th ECCMID, Madrid 2018.

14. Rao K, Micic D, Natarajan M et al. Clostridium difficile Ribotype 027: Relationship to Age, Detectability of Toxins A or B in Stool With Rapid Testing, Severe Infection, and Mortality. Clin Infect Dis 2015; 61 (2): 233-241.

15. Dudakova L, Galova Z, Melter O. Genotyping of Clostridium difficile from patients with suspect infection cdad (Clostridium difficile associated disease). MendelNet'09 Agro conference, Brno 2009: 629-637.

16. Freeman J, Vernon J, Pilling $S$ et al. The ClosER study: results from a three-year pan-European longitudinal surveillance of antibiotic resistance among prevalent Clostridium difficile ribotypes, 2011-2014. Clin Microbiol Infect 2018; 24 (7): 724-731.

17. Debast MP, Bauer EJ, Kuijper SB. European Society of Clinical Microbiology and Infectious Diseases: update of the treatment guidance document for Clostridium difficile infection. Clin Microbiol Infect 2014; 20 Suppl 2: 1-26.

18. Walk ST, Micic D, Jain R et al. Clostridium difficile Ribotype Does Not Predict Severe Infection. Clin Infect Dis 2012 15; 55 (12): 1661-1668.

Received September 5, 2019. Accepted November 29, 2019 